\title{
Medulloblastoma stem cells: where development and cancer cross pathways
}

\author{
Branavan Manoranjan', Chitra Venugopal', Nicole McFarlane', Bradley W. Doble', Sandra E. Dunn², Katrin Scheinemann ${ }^{3}$ \\ and Sheila K. Singh ${ }^{1}$
}

Brain tumors are the leading cause of childhood cancer mortality, with medulloblastoma (MB) representing the most frequent malignant tumor. The recent molecular classification of MB has reconceptualized the heterogeneity that exists within pathological subtypes by giving context to the role of key developmental signaling pathways in MB pathogenesis. The identification of cancer stem cell (CSC) populations, termed brain tumor-initiating cells (BTICS), in MB has provided novel cellular targets for the study of these aberrantly activated signaling pathways, namely, Sonic hedgehog (Shh) and Wingless (Wnt), along with the identification of novel BTIC self-renewal pathways. In this review, we discuss recent evidence for the presence of a MB stem cell that drives tumorigenesis in this malignant childhood tumor. We focus on evidence from cerebellar development, the recent identification of BTICs, the presence of activated developmental signaling pathways in $\mathrm{MB}$, the role of epigenetic stem cell regulatory mechanisms, and how these developmental and epigenetic pathways may be targeted for novel therapeutic options.

B rain tumors are the leading cause of childhood cancer mortality, with medulloblastoma (MB) representing the most frequent malignant tumor. James Homer Wright identified MB as a distinct central nervous system tumor in 1910 (1). At the time, Wright believed MB to arise from restricted neuronal precursors termed "neuroblasts." In 1925, Percival Bailey and Harvey Cushing described the presence of glial and neuronal cells in $\mathrm{MB}$, suggesting the cell of origin to be of a more primitive embryonic neuroepithelial cell, which they termed "medulloblast," resulting in the conceptual link between neural stem cells (NSCs) and MB ontogeny (2). More recent molecular classifications of $\mathrm{MB}$ have reconceptualized the heterogeneity that exists within pathological subtypes by giving context to the role of key developmental signaling pathways in MB pathogenesis (3-7). This work has lent greater support to the presence of a primitive cerebellar stem or precursor cell of origin. Novel therapeutic modalities may capitalize on the differences between the normal and malignant cerebellar precursor cell to specifically target the MB stem cell or its niche. Such therapies may prevent current treatment toxicity and long-term sequelae experienced by standard-risk patients despite a 5 -y survivorship of $80 \%$, and may be used to further target those high-risk patients who remain refractory to current treatment (8). In this review, we discuss recent evidence for the presence of a MB stem cell that drives tumorigenesis in this malignant childhood brain tumor. We focus on evidence from cerebellar development, the recent identification of brain-tumor-initiating cells (BTICs), the presence of activated developmental signaling pathways in $\mathrm{MB}$, the role of epigenetic stem cell regulatory mechanisms, and how these developmental and epigenetic pathways may be targeted with novel therapeutic options.

\section{THE CANCER STEM CELL HYPOTHESIS: BRIDGING NSCS} AND BTICS

Based on similarities observed between teratocarcinomas and the developing fetus, pathologists Rudolph Virchow and Julius Cohnheim first introduced the notion of a primitive cell of origin in malignant tissue more than 150 y ago $(9,10)$. Since then, this observation has evolved to what is now termed the cancer stem cell (CSC) hypothesis. This hypothesis suggests that a relatively small fraction of tumor cells, termed CSCs, have the ability to proliferate and maintain tumor growth (11). This is in sharp contrast to all other cells of the bulk tumor, which are characterized by limited proliferative capacity and a more specified lineage potential. More specifically, a CSC maintains two key properties: self-renewal and differentiation. Self-renewal is defined as the ability of a parental cell to generate an identical daughter cell and a second cell of the same or different phenotype, whereas through the process of differentiation a CSC is able to give rise to the heterogeneous cell lineages that constitute the original tumor (11). In the recent past, such CSC populations (also termed tumor-initiating cells, and in the case of brain cancer, BTICs) have been identified in a number of hematopoietic and solid-tumor malignancies 
based on cell surface markers and stem cell assays, of which the generation of tumors in human-mouse xenograft models has become the gold standard. Consequently, the functional definition of a CSC has no implication on its cellular origin within a tumor, and therefore a CSC may represent a transformed tissue-specific stem, progenitor, or differentiated cell.

The discovery of multipotent, self-renewing NSCs within the adult mammalian brain has provided reasonable evidence to suggest that a normal NSC or progenitor cell may be the target for malignant transformation in brain tumors (12-14). A key property of NSCs that may regulate their oncogenic potential is self-renewal. Given that normal NSCs must self-renew and maintain a relative balance between self-renewal and differentiation, brain tumorigenesis may be conceptualized as a disease of dysregulated self-renewal. Additional functional and genetic similarities shared by NSCs and brain tumor cells have been observed in histological studies of morphologically primitive tumors such as MB. These studies have noted both the expression of nestin, a marker of neural precursor cells, and the absence of markers typically expressed in differentiated cells $(15,16)$. Moreover, the heterogeneity of brain tumors, characterized by the expression of multiple neural lineage phenotypes, provides further evidence for a multipotent cell of origin. Consequently, an NSC may be seen as a more permissive and likely target for transformation, as the capacity for long-term self-renewal may promote the accumulation of mutations in a clonal population. A progenitor cell may also be a plausible target, given that the genetic alteration enables the cell to reacquire its self-renewal machinery. It remains to be seen if the tumorigenic event occurs solely in an NSC or in a more differentiated cell type that has reacquired stem cell characteristics.

The concept of a CSC suggests that tumors are organized into a hierarchy with distinct clonal populations of cells with only the CSC demonstrating the properties of self-renewal and differentiation in vitro and in vivo (11). Using in vitro assays originally developed to purify NSCs (12-14), Singh et al. (17) reported the identification and purification of a cell from primary human $\mathrm{MB}$ that had a marked capacity for proliferation, self-renewal, and differentiation. The BTIC represented a minority of the tumor cell population and was marked by expression of the cell surface marker CD133. Additional in vivo characterization of the BTIC using a human-mouse xenograft assay formally established the identification of CSCs in brain tumors (18). This work was corroborated by similar findings in which $\mathrm{MB}$ stem-like cells were found to express high levels of the NSC genes CD133, Sox2, Musashi1, and Bmi1, providing credence to NSC-driven tumorigenesis in MB (19).

\section{THE DEVELOPING CEREBELLUM: A TARGET OF TRANSFORMATION?}

Although CSCs have been shown to exist in MB, an appreciation of normal cerebellar development is necessary to identify a potential target for transformation into a CSC phenotype. Cerebellar development begins at several regions, including the upper rhombic lip and the ventricular zone (VZ) surrounding the fourth ventricle, during embryonic growth and only reaches complete maturation several months after birth (20). This makes the cerebellum a vulnerable target for physical abnormalities and oncogenic mutations. The VZ consists of stem and progenitor cells that line the fourth ventricle and is important for the generation of many cell types, including Purkinje neurons, interneurons, and glial cells. In contrast, progenitors within the upper rhombic lip give rise to all granule lineage cells of the external and internal granule layers (EGL and IGL, respectively). During fetal development, granule neuron precursors (GNPs) that cover the cerebellar surface divide in the EGL and then exit the cell cycle and migrate inward past the Purkinje cell layer to the IGL, where they become postmitotic granule neurons (21). In addition to the VZ stem and EGL progenitor cell populations, other cerebellar precursor cells have been described that could potentially initiate MB. Lee et al. (22) reported the in vitro and in vivo characterization of $\mathrm{CD} 133^{+}$stem cells concentrated in the white matter of the normal postnatal cerebellum. Using the markers Pax6/Tbr2/Tbr1 and Math1, respectively, both Fink et al. (23) and Wang et al. (24) have also identified novel precursor cells in the upper rhombic lip capable of generating nuclei in the deep cerebellar white matter.

In their initial description of $\mathrm{MB}$, Bailey and Cushing proposed the medulloblast to originate from a VZ stem cell (2). Subsequent immunohistochemical evidence suggested a dual-origin hypothesis in which MBs of different histological subtypes may result from a VZ stem or EGL progenitor cell. Desmoplastic MBs expressed markers associated with EGLderived GNPs such as Zicl (25) and Math1 (26), whereas classic MBs expressed markers of VZ-derived progenitors such as Calbindin (27) and glial fibrillary acidic protein (GFAP) (28). Therefore, classic MBs were thought to originate from the VZ, whereas GNPs in the EGL were believed to give rise to desmoplastic MBs. Gene expression profiling of these two histological subtypes corroborated the findings for desmoplastic MBs as they expressed genes associated with proliferating GNPs in the EGL, but classic MBs were found to express genes not specific to a particular cerebellar cell type $(6,29)$. Additional microarray analyses have failed to find clear correlations between lineage markers and histological subtypes (30), and with the identification of high levels of putative stem cells, including the VZ-associated CD133 ${ }^{+}$MB BTICs in both subtypes (19), it has become apparent that both histological subtypes of MB contain cells that resemble multipotent NSCs. Although the true origin remains unknown, recent molecular classifications have provided reasonable evidence for GNPs and brainstem precursors as potential cellular origins for MB.

\section{MOLECULAR PROFILING OF MB}

Although previous classifications based on histological subtypes and cerebellar precursor cell marker expression were unsuccessful at identifying a CSC origin, the recent classification of $\mathrm{MB}$ based on multiple genomic platforms has reconceptualized the heterogeneity that exists within pathological subtypes while also giving context to the role of key developmental signaling 
pathways in regulating BTICs (see schematic, Figure 1) (3-7). This new molecular classification system consists of several subtypes, each distinct in terms of prognosis and predicted therapeutic response. Unsupervised hierarchical clustering methods of segregating these data have produced four or five subgroups, two of which (subtypes A and B) are characterized by upregulation of genes in the Wingless (Wnt) or Sonic hedgehog (Shh) pathways, respectively. These two subgroups are separated from each other and other subgroups on principal components analysis and both are associated with improved clinical outcomes as compared with subtypes $\mathrm{C}, \mathrm{D}$, and $\mathrm{E}$, which are characterized by a greater propensity for metastatic disease and poor clinical outcomes (3-5,31-33). These aggressive MBs are collectively known as "non-Shh/Wnt" subtypes, which remain refractory to current treatment modalities and are without aberrant activation of specific signaling pathways, possibly suggesting the involvement of novel BTIC self-renewal genes and pathways (Figure 1) (34).

\section{Shh Subtype MB}

In normal development, Hedgehog (Hh) functions as a morphogen to induce cell identities in the ventral spinal cord and as a mitogen to drive proliferation of GNPs (35). The secreted Shh ligand, when bound to its cognate receptor Patched (Ptch1), activates the constitutively repressed Smoothened (Smo) receptor, which in turn activates the glioma-associated oncogene (Gli) family of transcription factors, Gli1, Gli2, and Gli3. Activation of Gli2 and Gli3 by Shh signaling suppresses the cleavage of Gli3 into its truncated repressor form, allowing both Gli2 and Gli3 to induce transcription of target genes such as Gli1. Gli1 expression subsequently leads to a positive feed-forward mechanism inducing Gli1-specific genes, many of which are essential regulators of self-renewal in both development and cancer $(36,37)$.

In normal cerebellar development, Shh is produced by Purkinje cells and binds to Ptch1, inducing the proliferation of GNPs by relieving the inhibition of Smo $(20,21,38)$. Shh signaling was first implicated in $\mathrm{MB}$ formation following the discovery of inactivating Ptch1 mutations in the germline of patients with nevoid basal cell carcinoma syndrome (also known as Gorlin syndrome), who have a MB prevalence of $1-2 \%(39,40)$. Genomic alterations in components of the Shh signaling pathway have been identified in up to $25 \%$ of sporadic human MBs and consist of inactivating mutations of Ptch 1 and Suppressor of fused (Sufu) and/or activating mutations of Smo (41-45).

Murine models, particularly transgenic mice haploinsufficient for Ptch1 $\left(P t c h 1^{+/-}\right)$, have greatly contributed to elucidating the role of Shh signaling in MB pathogenesis (41). Ptch1 haploinsufficiency increases the proliferation of NSCs, resulting in $\mathrm{MB}$ in $15-20 \%$ of $\mathrm{Ptch}^{+/-}$mice $(41,46,47)$. Initial work with this model identified cells resembling GNPs from the EGL that retained their proliferative potential, suggesting that GNPs promote Shh-driven MB (41). Subsequent gene expression profiles of $P t c h 1^{+/-}$tumors have demonstrated the overexpression of important targets in GNP proliferation (48). Most recently, both Schuller et al. (49) and Yang et al. (50) determined that dysregulated Shh signaling in unipotent GNPs or multilineage embryonic central nervous system stem cells is

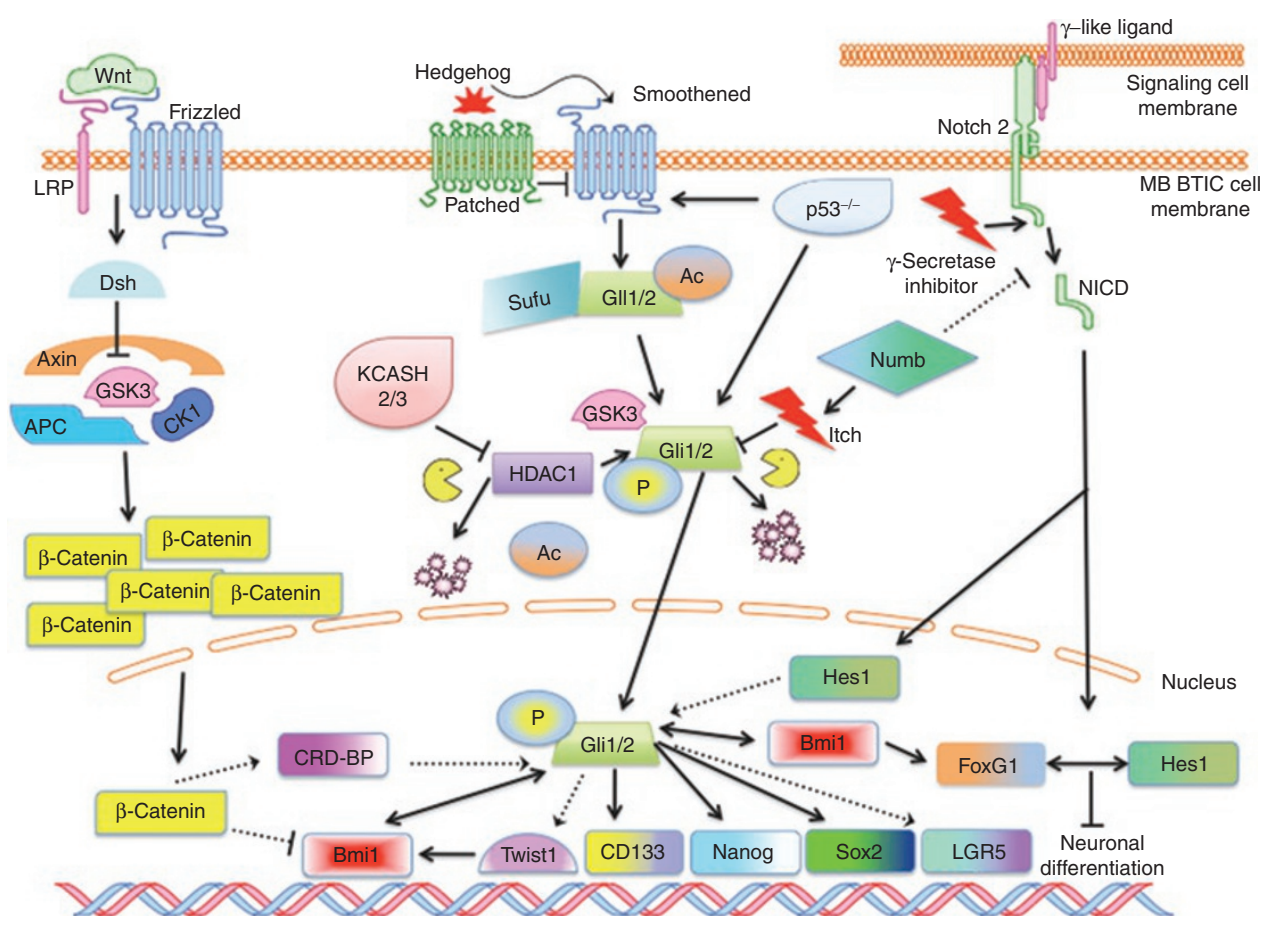

Figure 1. Activation of key developmental signaling pathways in MB. The Shh, Wnt, and Notch signaling pathways have been implicated in maintaining the tumorigenic potential of MB BTICs. Through interacting with mediators of Bmi1 signaling and other putative BTIC self-renewal genes, these novel regulatory mechanisms may promote or inhibit BTIC self-renewal and thereby provide avenues for therapeutic interventions. BTIC, brain tumor-initiating cell; MB, medulloblastoma; Shh, Sonic hedgehog; Wnt, Wingless. 
capable of generating $\mathrm{MB}$ following differentiation into the granule cell lineage. This work has implicated the acquisition of a GNP phenotype to be a critical stage in oncogenesis for Shh-driven MB.

Although a GNP may very well serve as the cell of origin for the Shh subtype of MB, the identification of tumor-propagating cells within this subtype is required for targeted therapeutic interventions. Of note, Read et al. (46) found CD15 to be a marker of BTIC populations in MB derived from $\mathrm{Ptch}^{+/-}$ mice. $\mathrm{CD} 15^{+}$cells constituted a small fraction of normal GNPs (as indicated by the coexpression of Math1), and MB cells exhibited a higher proliferative capacity and elevated levels of the Shh target genes Gli1 and Cyclin D1 as compared with $\mathrm{CD}^{-} 5^{-}$cells. However, $\mathrm{CD} 15^{+}$cells did not display multilineage differentiation or neurosphere formation when cultured at clonal densities per the characterization of CD133 as a BTIC marker. Consequently, it was believed that these cells marked progenitor populations as opposed to a more primitive stemlike cell. However, Ward et al. (47) demonstrated the propagation of multipotent $\mathrm{CD} 15^{+}$BTICs from $P t c h 1^{+/-}$mouse MB under stem-like serum-free conditions, and therefore suggested that CD15 marks a more primitive stem-like cell. Although the evidence for a GNP origin as the BTIC for Shh subtype MB is paramount, it remains unclear as to how these cells propagate and transform over the course of tumorigenesis as defined by their regulatory mechanisms and marker expression.

\section{Wnt Subtype MB}

The canonical Wnt pathway is mediated by $\beta$-catenin, a cytoplasmic protein whose stability is regulated by a multiprotein destruction complex consisting of Axin, adenomatous polyposis coli (APC), glycogen synthase kinase 3 (GSK3), and casein kinase 1 (CK1). In the absence of Wnt stimulation, $\beta$-catenin is phosphorylated by CK1 and GSK3, resulting in the ubiquitination and subsequent degradation of $\beta$-catenin. Activation of the Wnt pathway though the binding of Wnt ligands to the family of cell surface Frizzled receptors results in an intracellular cascade allowing $\beta$-catenin to accumulate in the cytosol by evading phosphorylation and degradation. Through mechanisms that remain unknown, the unphosphorylated $\beta$-catenin translocates to the nucleus, where it acts as a transcriptional coactivator by interacting with a family of transcription factors known as T-cell factors/lymphoid enhancer factors (TCF/ LEFs). In the absence of a Wnt signal, the TCF/LEFs form a complex with Groucho and histone acetylases to repress Wnt target genes. However, the binding of $\beta$-catenin to TCF/LEF alleviates the repressive activity of Groucho, activating TCF target genes such as $c-m y c$ and Cyclin D1 (51).

During embryonic growth, activation of the canonical Wnt signaling pathway plays an important role in regulating NSC proliferation and defining the midbrain-hindbrain boundary from which the entire cerebellum develops (52). Similar to the characterization of the Shh signaling pathway in MB, Wnt signaling was first implicated in the pathogenesis of $\mathrm{MB}$ by its activation in a familial cancer syndrome-Turcot syndrome (53). Mutations in several members of the Wnt pathway have since been identified in $\mathrm{MB}$, accounting for $25 \%$ of sporadic $\mathrm{MB}(5,31-33)$. These include activating mutations in CTNNB1 (which codes for $\beta$-catenin) and inactivating mutations in $A P C$ and Axin (54-56). Of interest, such mutations characteristic of Wnt subtype MB patients are associated with an improved survivorship as compared with all other subtypes, including Shh-driven MB (31-33). The first mouse model of the human Wnt MB subgroup was created in the past year; Gibson et al. (57) identified genes marking the human Wnt subtype to be more frequently expressed in the lower rhombic lip and embryonic dorsal brainstem than the upper rhombic lip. Having identified a distinct germinal zone of the hindbrain located in the lower rhombic lip, the group hypothesized Wnt subtype MB to originate from cells outside the cerebellum in the dorsal brainstem. Future efforts should assess the BTIC phenotype of these putative cells of origin in terms of their self-renewal capacity, differentiation potential, and in the case of Wnt-driven MB, susceptibility to chemotherapeutic agents.

\section{Non-Shh/Wnt Subtype MB}

With the plethora of data on Shh-driven MB and recent identification of putative cells of origin in a murine model of the Wnt subtype, current focus must shift to those non-Shh/Wnt subtype patients who are characterized by metastasis, remain refractory to therapy, and lack aberrant activation of known signaling pathways. The chromatin-modifying Polycomb-group $(\mathrm{PcG})$ gene Bmil has been identified as a critical regulator of NSC self-renewal through repression of the $\mathrm{p} 16^{\text {Ink4a }}$ and $\mathrm{p} 19^{\text {Arf }}$ senescence pathways (58). The Bmil signaling pathway is also consistently dysregulated or overexpressed in several emerging CSC populations, most recently being cited as a marker of recurrence, poor treatment response, metastatic potential, and death in many cancer models, including MB $(59,60)$.

As a PcG gene, Bmil is believed to function as a critical epigenetic regulator of fate determination in normal and CSC populations (61). Having been implicated in MB pathogenesis in conjunction with the activation of the Shh signaling pathway, previous reports have suggested a novel regulatory mechanism whereby an external signaling morphogen interacts with cell-intrinsic epigenetic pathways controlling cell fate programs $(62,63)$. Wang et al. (64) formally established this link between Shh signaling and Bmil expression, determining that Shh induces Bmil expression in human $\mathrm{MB}$ BTICs through the preferential binding of the Shh target Gli1 at the Bmil promoter. This work also demonstrated the presence of a feedback mechanism whereby downstream effectors of Bmil may activate Shh pathway genes, implicating Bmil and Shh as mutually indispensible pathways in MB BTIC self-renewal and maintenance. Of note, Bmil is preferentially overexpressed in non-Shh/Wnt subtype MB (64), providing a novel mechanism in which downstream effectors of Bmil may converge on additional signaling pathways, resulting in non-Shh/Wnt-driven MB.

Notch signaling is another pathway that may interact with downstream effectors of Bmil in non-Shh/Wnt MB. Notch2 
is predominantly expressed in proliferating GNPs during cerebellar development and is overexpressed in a subset of MB patients (65). Induction of the Notch pathway results in the activation of the transcription factor Hes1 (66), which is associated with poor survival in a subset of MB patients (65). Hes 1 expression has recently been hypothesized to form a transcriptional repressor complex with FoxG1 to negatively regulate NSC differentiation (67). FoxG1, a known Bmil downstream target, is a forebrain-specific transcription factor that is frequently dysregulated in $\mathrm{MB}$ and involved in the maintenance of NSC multipotency and self-renewal through a Bmil-dependent mechanism $(67,68)$. Given that Notch signaling has been shown to not regulate Shh-induced MB and thereby function independently (69), it may be hypothesized that non-Shh/Wnt MBs are driven by a number of mutually independent Bmil signaling pathways.

The identification of Twist 1 , a transcription factor, as an upstream target of Bmil that promotes the epithelialmesenchymal transition (EMT) in normal development and metastatic/invasive properties in cancer, has added yet another component to the Bmil signaling pathway (70). Twist 1 is also preferentially expressed in non-Shh/Wnt subtype MBs (64). Given the recent interest in the ability of CSCs to undergo EMT, the role of Twist 1 in promoting BTIC invasion and metastasis, characteristic of non-Shh/Wnt patients, may identify additional Bmil signaling pathways.

Aside from Bmil, p53 signaling has also been shown to regulate stem cell genes that may further characterize non$\mathrm{Sh} /$ Wnt subtype MB. The transcription factor Nanog is involved in embryonic stem cell self-renewal and reprogramming of somatic cells to induced pluripotent stem cells. Po et al. (71) have identified a novel mechanism through which the loss of $p 53$ activates the Shh targets Gli1 and Gli2 to bind Nanog-specific cis-regulatory sequences. The resulting pathway when initiated in mouse and human cerebellar stem cells results in the initiation and maintenance of $\mathrm{MB}$. Moreover, MBs derived from mice injected with $R b$ - and p53-inactivated NSCs, overexpress the stem cell markers nestin, Sox2, and Sox9. These markers have also been shown to identify a subset of human MB patients with poor clinical outcome (72).

Several mediators of NSC self-renewal, proliferation, and differentiation continue to be investigated with the intent that these regulators may assist in characterizing the subset of patients with $\mathrm{MB}$ who remain without curative therapies. Future efforts in identifying mechanisms of Wnt signaling that provide Wnt subtype patients with a drastically improved survivorship may also assist in elucidating novel pathways that remain dysregulated in patients with non-Shh/Wnt MB.

\section{TARGETING MB STEM CELLS}

Therapies targeted at BTICs are considered to deplete the oncogenic potential of the growing tumor because BTICs constitute a distinct clonal population with an enhanced proliferative potential and self-renewal capacity as compared with all other cell types in a brain tumor. However, BTICs are also thought to be resistant to current radiotherapy and chemotherapy, which may explain why some patients relapse despite current interventions. The aberrant activation of several signaling pathways in $\mathrm{MB}$ provides for countless therapeutic targets that may be harnessed to identify essential avenues for BTIC survival. Following radiation, MB BTICs have been found to activate PI3K/Akt signaling and undergo p53-dependent cell cycle arrest (73). This mechanism has enabled MB BTICs to reenter the cell cycle at a later time point to maintain their oncogenic effects. The ability of these cells to induce $p 53$ largely depends on the presence of phosphatase and tensin homolog (PTEN). Consequently, inhibition of Akt signaling has sensitized MB BTICs to radiationinduced apoptosis.

Inhibition of the Notch signaling pathway has also resulted in targeting MB BTICs. $\gamma$-Secretase inhibitors suppress the expression of Hes 1, resulting in a fivefold reduction of CD133+ BTICs (74). MicroRNAs have also been shown to block Notch signaling. miR-199b-5p has demonstrated inhibition of Hes 1, resulting in impaired engraftment of $\mathrm{MB}$ BTICs and a reduction in CD $133^{+}$BTICs (75). Clinical correlation of survival of patients with $\mathrm{MB}$ and $m i R-199 b-5 p$ expression identified a positive trend toward improved overall survivorship. Given that MB tumor burden was shown to be reduced in a xenograft model, experimental approaches with the use of $m i R-199 b-5 p$ as an adjuvant therapy in combination with current multimodal interventions may assist in improving the overall survivorship of $\mathrm{MB}$ patients. The interaction between Notch signaling and hypoxia-inducible factor $1 \alpha(H I F-1 \alpha)$ provides an additional MB BTIC therapeutic target. Inhibition of Notch 1 combined with the acute exposure of MB BTICs to $20 \%$ oxygen resulted in differentiation or apoptosis of MB BTICs (76). It is apparent that although the Notch pathway does not characterize a specific MB molecular subtype, it converges on several key pathways essential for BTIC survival.

The Shh pathway is by far the most studied in MB ontogeny and as expected consists of several novel targets. The finding that deletion of Math1, an essential transcription factor for Shh signaling in GNPs, prevents the development of Shhdriven $\mathrm{MB}$ has provided an interesting regulatory mechanism for MB formation (77). Most recently, Math1 has been shown to maintain GNPs in a Shh-responsive state by regulating genes that promote neuronal differentiation (78). The use of smallmolecule inhibitors directed at Math1 could remove its constitutive activation of GNPs to Shh and thereby prevent MB formation. Histone deacetylase 1 (HDAC1) has recently been shown to modulate Shh signaling through deacetylation of Gli1 and Gli2 and thereby enhance the transcriptional activity of these Shh effector proteins (79). Consequently, HDAC inhibitors may represent novel therapeutic agents targeted at Shh-mediated MB. The identification and characterization of a new family of HDAC inhibitor proteins termed "potassium channel tetramerization domain (KCTD) containing, Cullin3 adaptor, suppressor of Hedgehog" has demonstrated a remarkable reduction in the 
growth of Shh-driven $\mathrm{MB}$, suggesting the use of epigenetic regulators in preventing $\mathrm{MB}$ tumorigenesis (80). Although research efforts in targeting MB BTICs has primarily focused on the ability of novel agents to reduce the viability of $\mathrm{CD} 133^{+}$cells, Wang et al. (64) have proposed a cell-cell interaction model based on CD133 marker expression and Shh receptor phenotype in MB BTICs. CD $133^{+}$BTICs resembled GNPs during cerebellar development in that they contained the Shh signaling machinery and Shh receptors, whereas CD133- $\mathrm{MB}$ cells resembled Purkinje cells of normal development by serving as Shh-secreting cells. Consequently, it is imperative to also investigate the role of nonBTICs (i.e., CD133- cells) in MB tumorigenesis, as they may actually promote the survival of MB BTICs.

\section{CONCLUSIONS}

The study of MB tumorigenesis has drastically evolved over the past $100 \mathrm{y}$, with several key discoveries having been made only in the past decade. With the advent of molecular subtyping and deep genome sequencing of malignant tissue, the identification of additional classification systems rooted in clinical outcome and risk stratification is just around the corner. Nevertheless, the regulatory mechanisms within BTIC populations that initiate and maintain these tumors continue to serve as areas of particular interest for targeted therapeutic interventions. Novel BTIC self-renewal signaling pathways may enable the characterization of high-risk, poor-outcome patients who remain refractory to therapy.

\section{STATEMENT OF FINANCIAL SUPPORT}

This work was supported by funds from the Department of Surgery at McMaster University, the Ontario Institute for Cancer Research, and the JP Bickell Foundation. B.M. held training awards from the Stem Cell Network and Canadian Institutes of Health Research.

\section{REFERENCES}

1. Wright JH. Neurocytoma or neuroblastoma, a kind of tumor not generally recognized. J Exp Med 1910;12:556-61.

2. Bailey P, Cushing H. Medulloblastoma cerebelli: a common type of midcerebellar glioma of childhood. Arch Neurol Psychiatry 1925;14:192-223.

3. Cho YJ, Tsherniak A, Tamayo P, et al. Integrative genomic analysis of medulloblastoma identifies a molecular subgroup that drives poor clinical outcome. J Clin Oncol 2011;29:1424-30.

4. Kool M, Koster J, Bunt J, et al. Integrated genomics identifies five medulloblastoma subtypes with distinct genetic profiles, pathway signatures and clinicopathological features. PLoS ONE 2008;3:e3088.

5. Northcott PA, Korshunov A, Witt H, et al. Medulloblastoma comprises four distinct molecular variants. J Clin Oncol 2011;29:1408-14.

6. Pomeroy SL, Tamayo P, Gaasenbeek M, et al. Prediction of central nervous system embryonal tumour outcome based on gene expression. Nature 2002;415:436-42.

7. Thompson MC, Fuller C, Hogg TL, et al. Genomics identifies medulloblastoma subgroups that are enriched for specific genetic alterations. J Clin Oncol 2006;24:1924-31.

8. Gilbertson RJ. Medulloblastoma: signalling a change in treatment. Lancet Oncol 2004;5:209-18.

9. Virchow R. Editorial. Virchows Arch Pathol Anat Physiol Klin Med 1885;3:23.

10. Cohnheim J. (Ueber entzuendung und eiterung). Path Anat Physiol Klin Med 1867;40:1-79.

11. Clarke MF, Dick JE, Dirks PB, et al. Cancer stem cells-perspectives on current status and future directions: AACR Workshop on cancer stem cells. Cancer Res 2006;66:9339-44.
12. Reynolds BA, Weiss S. Generation of neurons and astrocytes from isolated cells of the adult mammalian central nervous system. Science 1992;255:1707-10.

13. Uchida N, Buck DW, He D, et al. Direct isolation of human central nervous system stem cells. Proc Natl Acad Sci USA 2000;97:14720-5.

14. Reynolds BA, Weiss S. Clonal and population analyses demonstrate that an EGF-responsive mammalian embryonic CNS precursor is a stem cell. Dev Biol 1996;175:1-13.

15. Dahlstrand J, Collins VP, Lendahl U. Expression of the class VI intermediate filament nestin in human central nervous system tumors. Cancer Res 1992;52:5334-41.

16. Lendahl U, Zimmerman LB, McKay RD. CNS stem cells express a new class of intermediate filament protein. Cell 1990;60:585-95.

17. Singh SK, Clarke ID, Terasaki M, et al. Identification of a cancer stem cell in human brain tumors. Cancer Res 2003;63:5821-8.

18. Singh SK, Hawkins C, Clarke ID, et al. Identification of human brain tumour initiating cells. Nature 2004;432:396-401.

19. Hemmati HD, Nakano I, Lazareff JA, et al. Cancerous stem cells can arise from pediatric brain tumors. Proc Natl Acad Sci USA 2003; 100:15178-83.

20. Wang VY, Zoghbi HY. Genetic regulation of cerebellar development. Nat Rev Neurosci 2001;2:484-91.

21. Sotelo C. Cellular and genetic regulation of the development of the cerebellar system. Prog Neurobiol 2004;72:295-339.

22. Lee A, Kessler JD, Read TA, et al. Isolation of neural stem cells from the postnatal cerebellum. Nat Neurosci 2005;8:723-9.

23. Fink AJ, Englund C, Daza RA, et al. Development of the deep cerebellar nuclei: transcription factors and cell migration from the rhombic lip. J Neurosci 2006;26:3066-76.

24. Wang VY, Rose MF, Zoghbi HY. Math1 expression redefines the rhombic lip derivatives and reveals novel lineages within the brainstem and cerebellum. Neuron 2005;48:31-43.

25. Yokota N, Aruga J, Takai S, et al. Predominant expression of human zic in cerebellar granule cell lineage and medulloblastoma. Cancer Res 1996;56:377-83.

26. Salsano E, Pollo B, Eoli M, Giordana MT, Finocchiaro G. Expression of MATH1, a marker of cerebellar granule cell progenitors, identifies different medulloblastoma sub-types. Neurosci Lett 2004;370:180-5.

27. Katsetos CD, Herman MM, Krishna L, et al. Calbindin-D28k in subsets of medulloblastomas and in the human medulloblastoma cell line D283 Med. Arch Pathol Lab Med 1995;119:734-43.

28. Kumanishi T, Washiyama K, Watabe K, Sekiguchi K. Glial fibrillary acidic protein in medulloblastomas. Acta Neuropathol 1985;67:1-5.

29. Kho AT, Zhao Q, Cai Z, et al. Conserved mechanisms across development and tumorigenesis revealed by a mouse development perspective of human cancers. Genes Dev 2004;18:629-40.

30. Lee Y, Miller HL, Jensen P, et al. A molecular fingerprint for medulloblastoma. Cancer Res 2003;63:5428-37.

31. Clifford SC, Lusher ME, Lindsey JC, et al. Wnt/Wingless pathway activation and chromosome 6 loss characterize a distinct molecular sub-group of medulloblastomas associated with a favorable prognosis. Cell Cycle 2006;5:2666-70.

32. Ellison DW, Onilude OE, Lindsey JC, et al.; United Kingdom Children's Cancer Study Group Brain Tumour Committee. beta-Catenin status predicts a favorable outcome in childhood medulloblastoma: the United Kingdom Children's Cancer Study Group Brain Tumour Committee. J Clin Oncol 2005;23:7951-7.

33. Fattet $\mathrm{S}$, Haberler $\mathrm{C}$, Legoix $\mathrm{P}$, et al. Beta-catenin status in paediatric medulloblastomas: correlation of immunohistochemical expression with mutational status, genetic profiles, and clinical characteristics. J Pathol 2009;218:86-94.

34. Ellison DW. Childhood medulloblastoma: novel approaches to the classification of a heterogeneous disease. Acta Neuropathol 2010; 120:305-16.

35. Fuccillo M, Joyner AL, Fishell G. Morphogen to mitogen: the multiple roles of hedgehog signalling in vertebrate neural development. Nat Rev Neurosci 2006;7:772-83. 
36. Lee EY, Ji H, Ouyang Z, et al. Hedgehog pathway-regulated gene networks in cerebellum development and tumorigenesis. Proc Natl Acad Sci USA 2010;107:9736-41.

37. Stecca B, Ruiz i Altaba A. Brain as a paradigm of organ growth: Hedgehog-Gli signaling in neural stem cells and brain tumors. J Neurobiol 2005;64:476-90.

38. Wechsler-Reya RJ, Scott MP. Control of neuronal precursor proliferation in the cerebellum by Sonic Hedgehog. Neuron 1999;22:103-14.

39. Gailani MR, Bale SJ, Leffell DJ, et al. Developmental defects in Gorlin syndrome related to a putative tumor suppressor gene on chromosome 9. Cell 1992;69:111-7.

40. Hahn H, Wicking C, Zaphiropoulous PG, et al. Mutations of the human homolog of Drosophila patched in the nevoid basal cell carcinoma syndrome. Cell 1996;85:841-51.

41. Goodrich LV, Milenkovic L, Higgins KM, Scott MP. Altered neural cell fates and medulloblastoma in mouse patched mutants. Science 1997;277:1109-13.

42. Hallahan AR, Pritchard JI, Hansen S, et al. The SmoAl mouse model reveals that notch signaling is critical for the growth and survival of sonic hedgehog-induced medulloblastomas. Cancer Res 2004;64:7794-800.

43. Oliver TG, Read TA, Kessler JD, et al. Loss of patched and disruption of granule cell development in a pre-neoplastic stage of medulloblastoma. Development 2005;132:2425-39.

44. Raffel C, Jenkins RB, Frederick L, et al. Sporadic medulloblastomas contain PTCH mutations. Cancer Res 1997;57:842-5.

45. Taylor MD, Liu L, Raffel C, et al. Mutations in SUFU predispose to medulloblastoma. Nat Genet 2002;31:306-10.

46. Read TA, Fogarty MP, Markant SL, et al. Identification of CD15 as a marker for tumor-propagating cells in a mouse model of medulloblastoma. Cancer Cell 2009;15:135-47.

47. Ward RJ, Lee L, Graham K, et al. Multipotent CD15+ cancer stem cells in patched-1-deficient mouse medulloblastoma. Cancer Res 2009;69:4682-90.

48. Kenney AM, Cole MD, Rowitch DH. Nmyc upregulation by sonic hedgehog signaling promotes proliferation in developing cerebellar granule neuron precursors. Development 2003;130:15-28.

49. Schüller U, Heine VM, Mao J, et al. Acquisition of granule neuron precursor identity is a critical determinant of progenitor cell competence to form Shh-induced medulloblastoma. Cancer Cell 2008;14:123-34.

50. Yang ZJ, Ellis T, Markant SL, et al. Medulloblastoma can be initiated by deletion of Patched in lineage-restricted progenitors or stem cells. Cancer Cell 2008;14:135-45.

51. Clevers H. Wnt/beta-catenin signaling in development and disease. Cell 2006;127:469-80.

52. Thomas KR, Capecchi MR. Targeted disruption of the murine int-1 proto-oncogene resulting in severe abnormalities in midbrain and cerebellar development. Nature 1990;346:847-50.

53. Hamilton SR, Liu B, Parsons RE, et al. The molecular basis of Turcot's syndrome. N Engl J Med 1995;332:839-47.

54. Baeza N, Masuoka J, Kleihues P, Ohgaki H. AXIN1 mutations but not deletions in cerebellar medulloblastomas. Oncogene 2003;22:632-6.

55. Eberhart CG, Tihan T, Burger PC. Nuclear localization and mutation of beta-catenin in medulloblastomas. J Neuropathol Exp Neurol 2000;59:333-7.

56. Huang H, Mahler-Araujo BM, Sankila A, et al. APC mutations in sporadic medulloblastomas. Am J Pathol 2000;156:433-7.

57. Gibson P, Tong Y, Robinson G, et al. Subtypes of medulloblastoma have distinct developmental origins. Nature 2010;468:1095-9.

58. Bruggeman SW, Valk-Lingbeek ME, van der Stoop PP, et al. Ink4a and Arf differentially affect cell proliferation and neural stem cell self-renewal in Bmil-deficient mice. Genes Dev 2005;19:1438-43.

59. Glinsky GV, Berezovska O, Glinskii AB. Microarray analysis identifies a death-from-cancer signature predicting therapy failure in patients with multiple types of cancer. J Clin Invest 2005;115:1503-21.
60. Zakrzewska M, Zakrzewski K, Grešner SM, Piaskowski S, ZalewskaSzewczyk B, Liberski PP. Polycomb genes expression as a predictor of poor clinical outcome in children with medulloblastoma. Childs Nerv Syst 2011;27:79-86.

61. Valk-Lingbeek ME, Bruggeman SW, van Lohuizen M. Stem cells and cancer; the polycomb connection. Cell 2004;118:409-18.

62. Leung $\mathrm{C}$, Lingbeek $\mathrm{M}$, Shakhova $\mathrm{O}$, et al. Bmi1 is essential for cerebellar development and is overexpressed in human medulloblastomas. Nature 2004;428:337-41.

63. Michael LE, Westerman BA, Ermilov AN, et al. Bmil is required for Hedgehog pathway-driven medulloblastoma expansion. Neoplasia 2008; 10:1343-9, 5p following 1349.

64. Wang X, Venugopal C, Manoranjan B, et al. Sonic hedgehog regulates Bmil in human medulloblastoma brain tumor-initiating cells. Oncogene 2011;31:187-99.

65. Fan X, Mikolaenko I, Elhassan I, et al. Notch1 and notch2 have opposite effects on embryonal brain tumor growth. Cancer Res 2004;64: 7787-93.

66. Pierfelice TJ, Schreck KC, Eberhart CG, Gaiano N. Notch, neural stem cells, and brain tumors. Cold Spring Harb Symp Quant Biol 2008;73: $367-75$.

67. Adesina AM, Nguyen Y, Mehta V, et al. FOXG1 dysregulation is a frequent event in medulloblastoma. J Neurooncol 2007;85:111-22.

68. Fasano CA, Phoenix TN, Kokovay E, et al. Bmi-1 cooperates with Foxg1 to maintain neural stem cell self-renewal in the forebrain. Genes Dev 2009;23:561-74

69. Julian E, Dave RK, Robson JP, Hallahan AR, Wainwright BJ. Canonical Notch signaling is not required for the growth of Hedgehog pathway-induced medulloblastoma. Oncogene 2010;29:3465-76.

70. Yang MH, Hsu DS, Wang HW, et al. Bmil is essential in Twist1-induced epithelial-mesenchymal transition. Nat Cell Biol 2010;12:982-92.

71. Po A, Ferretti E, Miele E, et al. Hedgehog controls neural stem cells through p53-independent regulation of Nanog. EMBO J 2010;29: 2646-58.

72. Sutter R, Shakhova O, Bhagat $\mathrm{H}$, et al. Cerebellar stem cells act as medulloblastoma-initiating cells in a mouse model and a neural stem cell signature characterizes a subset of human medulloblastomas. Oncogene 2010;29:1845-56.

73. Hambardzumyan D, Becher OJ, Rosenblum MK, Pandolfi PP, ManovaTodorova K, Holland EC. PI3K pathway regulates survival of cancer stem cells residing in the perivascular niche following radiation in medulloblastoma in vivo. Genes Dev 2008;22:436-48.

74. Fan X, Matsui W, Khaki L, et al. Notch pathway inhibition depletes stemlike cells and blocks engraftment in embryonal brain tumors. Cancer Res 2006;66:7445-52.

75. Garzia L, Andolfo I, Cusanelli E, et al. MicroRNA-199b-5p impairs cancer stem cells through negative regulation of HES1 in medulloblastoma. PLoS ONE 2009;4:e4998.

76. Pistollato F, Rampazzo E, Persano L, et al. Interaction of hypoxia-inducible factor-1a and Notch signaling regulates medulloblastoma precursor proliferation and fate. Stem Cells 2010;28:1918-29.

77. Flora A, Klisch TJ, Schuster G, Zoghbi HY. Deletion of Atoh1 disrupts Sonic Hedgehog signaling in the developing cerebellum and prevents medulloblastoma. Science 2009;326:1424-7.

78. Ayrault O, Zhao H, Zindy F, Qu C, Sherr CJ, Roussel MF. Atoh1 inhibits neuronal differentiation and collaborates with Gli1 to generate medulloblastoma-initiating cells. Cancer Res 2010;70:5618-27.

79. Canettieri G, Di Marcotullio L, Greco A, et al. Histone deacetylase and Cullin3-REN(KCTD11) ubiquitin ligase interplay regulates Hedgehog signalling through Gli acetylation. Nat Cell Biol 2010;12:132-42.

80. De Smaele E, Di Marcotullio L, Moretti M, et al. Identification and characterization of KCASH2 and KCASH3, 2 novel Cullin3 adaptors suppressing histone deacetylase and Hedgehog activity in medulloblastoma. Neoplasia 2011;13:374-85 